\title{
A Channel Simulator for Indoor Power-line Communications
}

\author{
S. Sancha, F. J. Cañete, L. Díez and J.T. Entrambasaguas \\ Dpto. Ingeniería de Comunicaciones \\ Universidad de Málaga (Spain)
}

\begin{abstract}
In this paper, a simulator for indoor power-line channels is presented. Its behavioral model includes both the long- and short-time variation of the channel. The long-time variation is due to the electrical appliances switching and is modeled in a statistical way. The cyclic short-time variation is related to the presence of the mains voltage and is characterized modeling the channel response as a linear periodically timevariant (LPTV) system and including an additive cyclostationary noise term. The cyclic model is simplified by using a slow variation approach, so that the channel can be modeled by means of a cyclic sequence of LTI systems with stationary noise terms. Channels according to the behavioral model are generated based on the physical structure of the network. The model for this physical structure comprises: the wiring, characterized as a set of terminated transmission lines connected in a tree-like fashion; and the electrical appliances, which are modeled as electric bipoles acting as impedances and noise sources. The simulator can perform the calculation of the channel frequency response and noise power spectral density at the receiver given a network topology. Afterwards, a channel simulation can be carried out using two banks of filters, one for the LPTV channel response and the other to obtain cyclostationary noise by filtering AWGN. Some test results for the channel generation and simulation are presented, and practical applications of this simulator are discussed.
\end{abstract}

Index Terms-Power-Line Communications (PLC), Channel Modeling, LPTV System, Cyclostationary Noise, Channel Simulation

\section{INTRODUCTION}

$\mathbf{P}$ OWER Line Communications (PLC) constitute nowadays a mature technology for local area networks at small offices and homes. Recently, triple-play solutions are becoming more popular and there is an increasing need for high quality broadband in-home communications. In this context, indoor PLC systems have proven to be not only a good solution themselves but also a valuable companion to other technologies, such as wireless, digital subscriber line or cable systems.

Indoor power-line channels exhibit variation with time at various levels [1]. On the one hand, there exits a long-time variation due to the switching of the electrical appliances, which is related to human activity and, therefore, random. On the other, a short-time variation appears as a result of the dependence on the mains voltage that these devices show. This twofold dependence complicates the modeling.

A typical power network in a house or small office may consist of four to six electric circuits that branch from the service panel to the sockets in a tree-like topology. Sockets may be in open circuit or have a load connected to them whose impedance, in general, will not be matched to the line impedance at high frequency. The appliances that can be found are of very varied nature, both in their electric behaviorimpedance they show to the network and noise they generateand in the way they are used.

Regarding to the noise at the receiver, we find noise generated by the electric appliances, which we call internal noise, and noise coming from coupled radio waves and from the rest of the electrical network [2], [3]. According to its statistical properties, the noise can be classified into cyclostationary (in periods of several minutes or hours) [1], [4], [5] or impulsive noise (with interarrival time in the range of seconds and duration of microseconds) [6]. The former also includes a stationary term, representing the background noise. Impulsive noise not related to mains period, which is essentially caused by the connection and disconnection of devices, is not considered in this work.

Channel properties are extremely sensitive to the network topology, the nature and switching state of the connected devices, and the sockets where the transmitter and receiver equipments are connected to. Besides, the high branching level of the network gives rise to many reflections (a phenomenon known as multipath propagation) that induce signal fading at several frequencies. All this makes it complicated to build a parametric channel model from real measurements. An alternative approach, which we apply here, is to use a realistic network structure model to generate channel behavior parameters [3].

The aim of the channel simulator we present in this paper is not to exactly reproduce the characteristics of a given power-line network, but to obtain plausible or expectable channel responses which can be used as a test-bench for new transmission techniques. With this target in mind, the capability to generate network topologies in a random fashion was implemented.

The organization of the paper is as follows. First, the mathematical model used to describe the channel both from a physical and a behavioral point of view is explained. Next, the channel generation procedure, which obtains the channel response from a given network topology, is described. Afterwards, we show how these results are used to simulate the channel performance. Finally, some test results are analyzed and we conclude discussing practical applications for this channel simulator. 


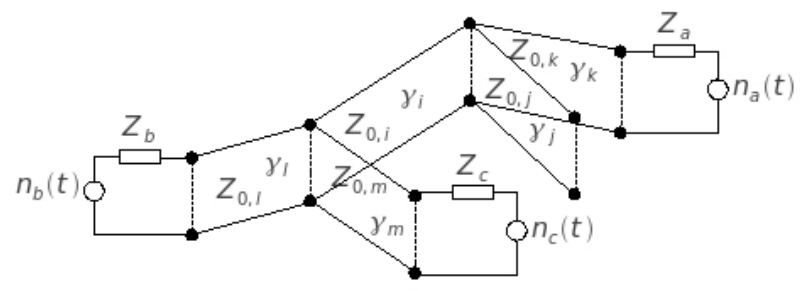

Fig. 1. Structural model

\section{DESCRIPTION OF THE MODEL}

The simulator uses a model of the network physical structure, upon which it builds a behavioral model. This approach allows to analyze the channel response sensitivity to the variation of a network element. Moreover, it shows advantage over a purely parametric model in the sense that making assumptions on the physical characteristics of an indoor electrical wiring is more intuitive than making them on its properties as a communication channel.

\section{A. Structural model}

The structural model comprises the wiring network and the electric appliances connected to it. The wiring is characterized as a set of transmission lines connected in a tree-like fashion. Each line is characterized by its length and the type of wire it is made of, which ultimately determines its characteristic impedance and propagation constant (Fig. 1).

The electric appliances connected to the network are modeled as electric bipoles with a twofold nature: impedances attached to the network and noise generators. Both their impedance and the power spectral density of the noise they generate will be a function of frequency and time in general (see more details in [7]). The restrictions we apply in that sense will determine the behavioral model.

\section{B. Behavioral model}

In the simulator two different behavioral models are used. The first one is based on the hypothesis of linearity and time invariance (LTI) of the electric devices, which allows us to model the channel as an LTI system. The second one takes into account the short-time variation of the loads related to the mains voltage.

1) LTI approach: The LTI model assumes that, in the shortterm, the impedance shown to the network by the appliances does not vary. Besides, the noise they generate is considered stationary.

The linearity and time invariance hypothesis has traditionally been applied to the power-line channel [8], [9]. Actually, what is assumed is that the only dependence on time comes from the connection and disconnection dynamics of the electric appliances, which is at a time scale that is several orders of magnitude over the one of the communication signal. This simplification is not justified in many situations, but the LTI model is a simple base upon which more complex models can be built.
The channel is modeled by means of an LTI system defined by its impulse response $h(t)$. The noise at the receiver is modeled as an additive random perturbation, characterized by a certain power spectral density (PSD).

2) LPTV approach: It has been verified that both the impedance the appliances show to the network and the power spectral density of the noise they generate suffer a cyclic variation, synchronized with the mains voltage. This variation comes from the devices' non-linear nature (e.g., they contain rectifiers). Nevertheless, from a small signal point of view, we are dealing with a linear, but periodically time-variant (LPTV), system [1], whose frequency impulse response is defined as

$$
H(t, f)=\int_{-\infty}^{\infty} h(t, t-\tau) \cdot e^{-j 2 \pi f \tau} d \tau
$$

where $h(t, t-\tau)$ is the impulse response which, as well as $H(t, f)$, is periodical in $t$.

Fortunately, a certain simplification can be applied by considering that the cyclic time variation of the channel is quite slow. It has been verified that the length of the impulse response is several orders of magnitude below the coherence time of the channel (the interval in which the channel properties can be considered invariant [10]). Under this slow variation hypothesis, the mains cycle can be divided into a number of invariance intervals, which give rise to a cyclic sequence of different LTI systems and stationary noise sources (see later the discrete-time case in Fig. 4). Note that the LTI model can be considered a particular case, with a single invariance interval.

\section{Long-time variation}

In the long term, electric appliances show a random variation due to their switching. Their electrical properties will be different in general when they are switched on and off, so these variations modify the channel response [7].

The state transitions of the loads are modeled as two-state homogeneous (with stationary transition probabilities) Markov processes. In a Markov process, the probability of moving from a state to other depends solely on the current state, and how long it has remained in it. Hence, the permanence time on a given state is an exponentially distributed random variable [11]. Each appliance is assigned an ON and OFF average permanence time, which defines its long-time behavior. Statistical values for these permanence times have been assigned according to the expected human behaviour. For instance, a microwave oven may have a 3 minutes permanence time in ON state, but for a TV set this time may be 60 minutes. State transitions are assumed to be instantaneous, and the time intervals among them are considered periods in which channel conditions are invariant.

\section{Time scales}

In the model three time scales, associated to different phenomena, are implicit (Fig. 2).

The random scale is related to the connection and disconnection of the electric appliances. It represents the long-time 


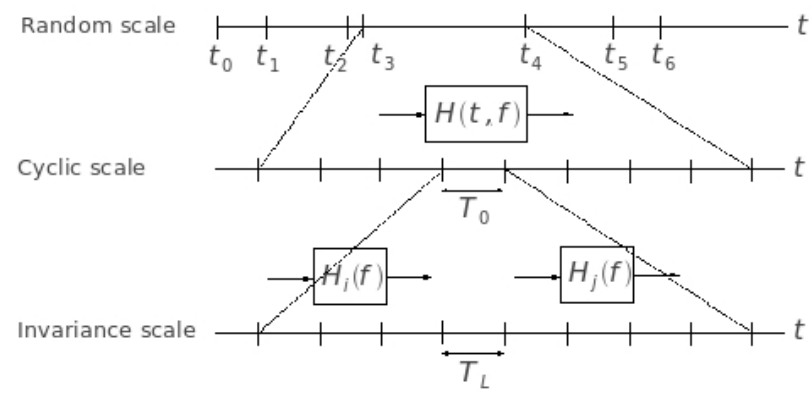

Fig. 2. Time scales

variation of the channel, and is in the range of minutes or even hours.

The cyclic scale corresponds to the cycles of the mains voltage, in which the channel is modeled as an LPTV system and the noise as having cyclostationary terms. All the intervals have the same length, $T_{0}=20 \mathrm{~ms}$ in a $50 \mathrm{~Hz}$ country, or $16.67 \mathrm{~ms}$ in a $60 \mathrm{~Hz}$ one.

The invariance scale is defined at the level of the $L$ subintervals the mains cycle is divided into, in which the channel is assumed to have an LTI behavior and the noise to have only stationary terms. All the intervals have the same length, $T_{L}=T_{0} / L$.

An appliance switching in the random scale gives rise to a new ensemble of the channel, which is modeled by means of $H(t, f)$, a certain LPTV system response, and $S_{N}(t, f)$, a noise instantaneous PSD [12]. The mains cycle is then divided into a series of intervals, indexed by $\ell \in[0, L-1]$, in which the channel is considered a snapshot with an LTI response $H_{\ell}(f)$ and the PSD of a stationary noise $S_{N, \ell}(f)$.

\section{ChanNEL Generation}

We call channel generation to the calculation of the channel frequency response and noise PSD at the receiver from a given network topology. It is therefore a direct application of the network structural model.

The LTI channel response $H_{\ell}(f)$ is obtained from the equivalent ABCD matrix of the main path between the transmitter and the receiver. The main path is the shortest sequence of lines that join those two nodes, and it determines the channel delay. The ABCD parameters associated to a given path are obtained by multiplying the matrices of the sections of line (in cascaded or bridged taps) that it contains. The tree branches that do not belong to the main path will act as bridged taps, whose equivalent impedance can be calculated recursively down to the terminal nodes (sockets). ABCD matrices values are calculated by straightforward application of basic transmission line theory [13].

The PSD of the noise at the receiver $S_{N, \ell}(f)$ can be calculated applying the same algorithm between every terminal node in which a noisy load is set and the receiver. Once we know the frequency response between those two nodes, the PSD of the noise generated by the load can be "filtered" to the receiver. To achieve this, a collection of impedance $Z_{\ell}(f)$ and

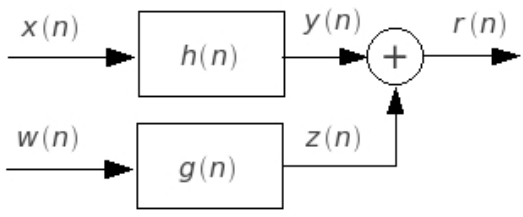

Fig. 3. LTI channel simulator

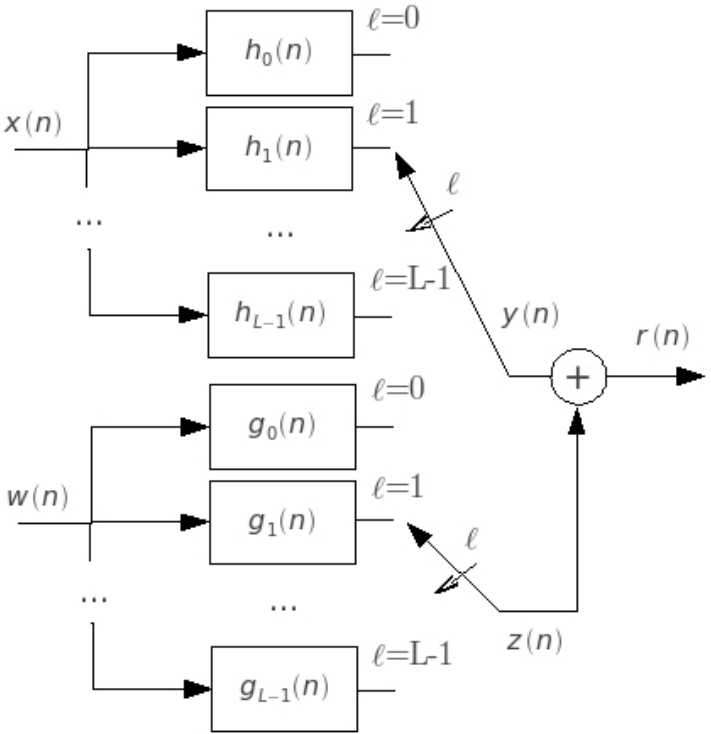

Fig. 4. LPTV channel simulator

noise $S_{N, \ell}(f)$ data has been obtained from real measurements of appliances [7]. These partial contributions, together with the external noise, are added to obtain the total noise.

\section{Channel Simulation}

The application of any of the two behavioral models lets us simulate the effect of the channel on an arbitrary signal $x(n)$. If we apply the invariant channel model, the simulator adopts the structure shown in Fig. 3, consisting of two LTI filters. The filter with impulse response $h(n)$, the channel filter, is calculated from the channel frequency response. The noise filter, with impulse response $g(n)$, colors a white Gaussian noise signal $w(n)$ (whose power is to be determined) to obtain a noise signal $z(n)$ with the desired PSD.

If the LPTV model is used, this structure extends to two banks of LTI filters which are applied in a cyclic manner, in which each filter corresponds to an invariance interval (Fig. 4).

\section{A. Channel filter}

The channel filter is implemented by means of a finite impulse response (FIR) structure. Its coefficients, which are its impulse response, can be calculated with the inverse discrete Fourier transform (IDFT) of the $H_{\ell}(f)$ that has been 
generated. This impulse response is usually shorter than the invariance interval $T_{L}$, so the number of coefficients can be reduced to minimize the computational complexity.

\section{B. Noise filter}

The design of the noise filter can be tackled as a random process modeling problem- synthesizing $z(n)$ by filtering a white Gaussian noise signal. Specifically, an auto-regressive (AR) model of the colored noise signal is used. This gives rise to an all-pole filter whose coefficients can be calculated solving the Yule-Walker equations. This can be efficiently accomplished using the Levinson-Durbin algorithm [14].

The autocorrelation sequence of the colored noise process, necessary to apply the algorithm, can be calculated by means of the IDFT of its power spectral density $S_{N, \ell}(f)$. The algorithm also returns the power of the white noise source.

\section{Interpolation order}

The LPTV simulator structure shown in Fig. 4 implies a zero-order interpolation on the impulse responses of two consecutive invariance intervals. If the channel variation is not slow enough (or a smaller number of invariance intervals is desired), so we cannot assume the impulse response in two consecutive intervals to be approximately equal, a firstorder interpolation can be applied in order to ensure smooth transitions (Fig. 5). That is, to interpolate linearly the response of two consecutive filters. In Fig. 5, $M$ is the number of samples per invariance interval; i.e., $M=\left\lfloor T_{L} / T_{s}\right\rfloor$, being $T_{s}$ the sampling period $(\lfloor x\rfloor$ means the integer part of $x)$. The interpolation index $\lambda$ ranges from 0 to $M-1$. Note that, both with zero-order and linear interpolation, the implementation of the LPTV channel simulator only requires processing the input of the filter whose output is being considered at that moment and the next one. Thus, the next filter memory is ready once the output is switched to it.

\section{Simulator OPERATION}

\section{A. Types of simulation}

The existence of two different behavioral models, as well as three time scales, gives rise to several sorts of simulations. Depending on the length of the simulation at the random scale we can consider static or dynamic simulations. In a static simulation the long-term variation of the channel is not taken into account, whereas in a dynamic simulation state transitions of the electric appliances are randomly generated, which gives rise to a sequence of static simulations.

Depending on the chosen behavioral model, the simulation can be variant or invariant. In a variant simulation, the channel periodicity is taken into account. In an invariant simulation this dependence is rejected, and the LTI model is assumed.

The dimension of the results of a channel generation, frequency response and PSD of the received noise, will depend on the type of simulation. In a static simulation, the results will be in the form of a vector- invariant case- or a set of vectorsvariant case- in $f$. In a dynamic one, they will be a matrix, or a set of matrices, in $(t, f)$.

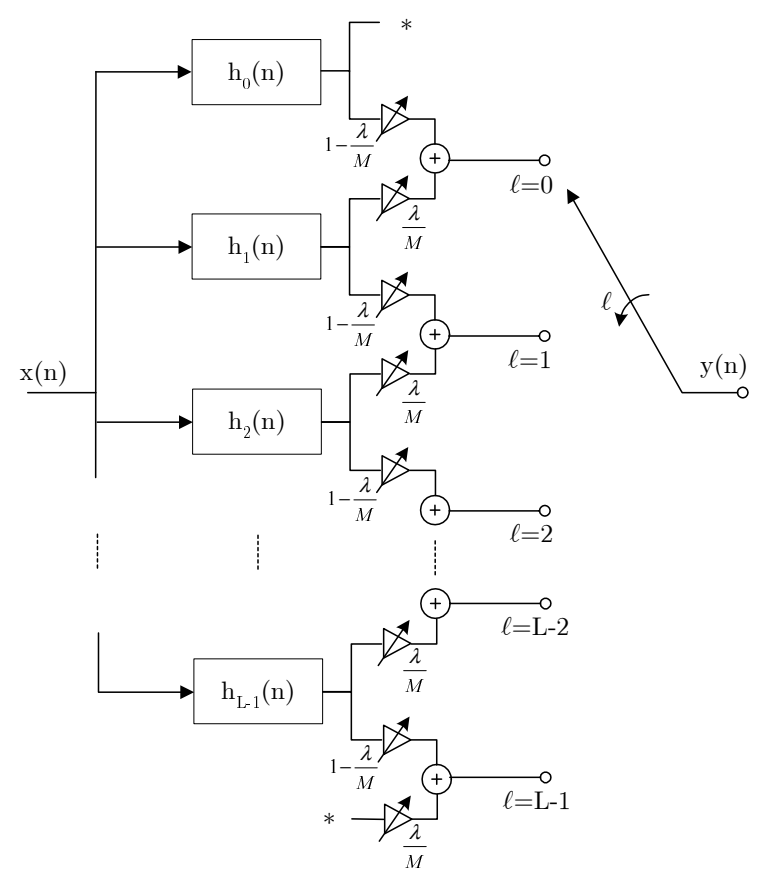

Fig. 5. Channel simulator using first-order interpolation

\section{B. Topology randomization}

The structure of the network (the topology tree), the length of the lines and/or the appliances connected to the terminal nodes can be determined at random, prior to the channel generation. Randomizing some or all of these parameters allows us to study the channel sensitivity to the physical characteristics of the network, as well as to the nature and distribution of the electric appliances connected to it.

\section{Simulator TESTS}

\section{A. Channel generation}

In order to illustrate the channel generation procedure a network topology was randomly generated. The resulting network consisted of 35 nodes, 34 lines and 6 different types of appliances connected to the terminal nodes (including the transmitter and receiver). Next, the LPTV behavioral model was chosen so the short-time variation of the loads was considered. The simulation length at the random scale was set to 0 , so the long-term variation of the channel was not simulated. That is, we obtained a snapshot of the cyclic channel at a given instant of the random scale. A detail of the generated structure, as seen in the simulator topology editor, is shown in Fig. 6.

The results of the channel generation are shown in Fig. 7 and 8. The channel frequency response (only amplitude) is plotted as a three-dimensional chart depending on both frequency and time. The latter spans through a mains cycle (20 ms), indexed by the $L$ invariance intervals. It is worth mentioning that a variation exceeding $20 \mathrm{~dB}$ appears at certain frequencies along the mains cycle. Otherwise, to plot the noise 


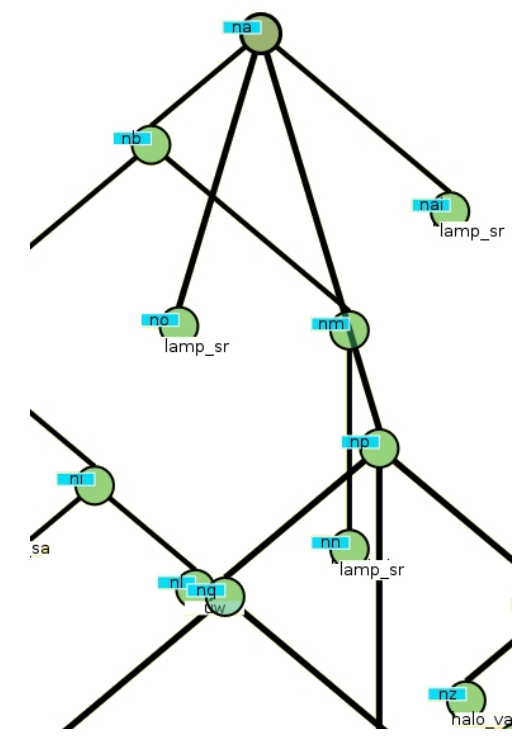

Fig. 6. Topology used in the channel generation test (detail)

PSD at the receiver an alternative approach is used: the curves corresponding to every invariance interval are superimposed in a bidimensional chart. In this case, a variation of noise level of more than $12 \mathrm{~dB}$ at lower frequencies or $5 \mathrm{~dB}$ at other ones is observed.

Fig. 9 and 10 show the results of a different type of simulation. This time, the LTI behavioral model was selected, neglecting the short-time variation of the channel. The length of the simulation was set to 120 minutes, so the long-time switching of the appliances was simulated. Each curve corresponds to a time interval in the random scale, delimited by the instants at which an appliance changed its state. These results exhibit as well remarkable variations of channel characteristics as time goes by.

\section{B. Channel simulation}

To test the channel simulation procedure we generated a 20ms-long input signal (a mains cycle) consisting of a sequence of impulses. The impulses were arranged along the signal length so they coincided with the instants at which an invariance interval started. That is,

$$
x(n)=\sum_{i=0}^{L-1} \delta\left(n-i\left\lfloor\frac{T_{L}}{T_{s}}\right\rfloor\right) \quad 0 \leq n \leq\left\lfloor\frac{T_{0}}{T_{s}}\right\rfloor
$$

being $L=292, T_{0}=20 \mathrm{~ms}$ and $T_{s}=1 / 60 \mathrm{MHz}$. Next, a channel was randomly generated applying the LPTV behavioral model, and the simulator was set up to emulate its response.

If we process $x(n)$ with the channel simulator, a matrix with $L$ impulse responses will be obtained. These responses will correspond to the $L$ LTI filters that represent each invariance interval, so by means of a discrete-time Fourier transform

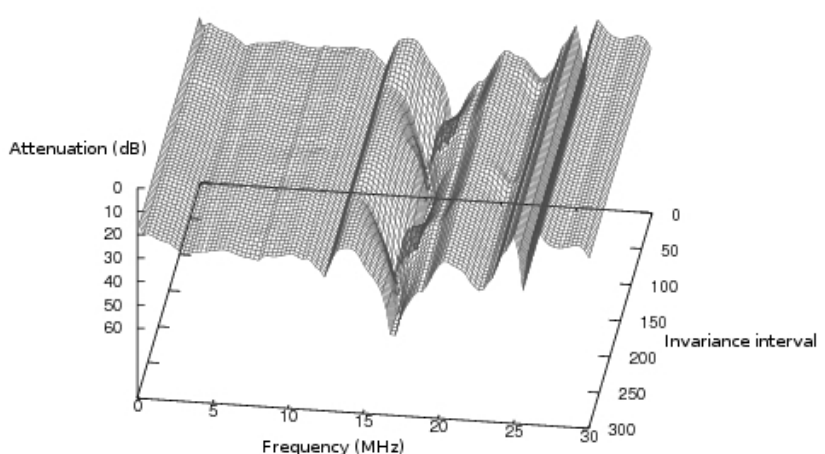

Fig. 7. Variant channel generation test results: channel response amplitude

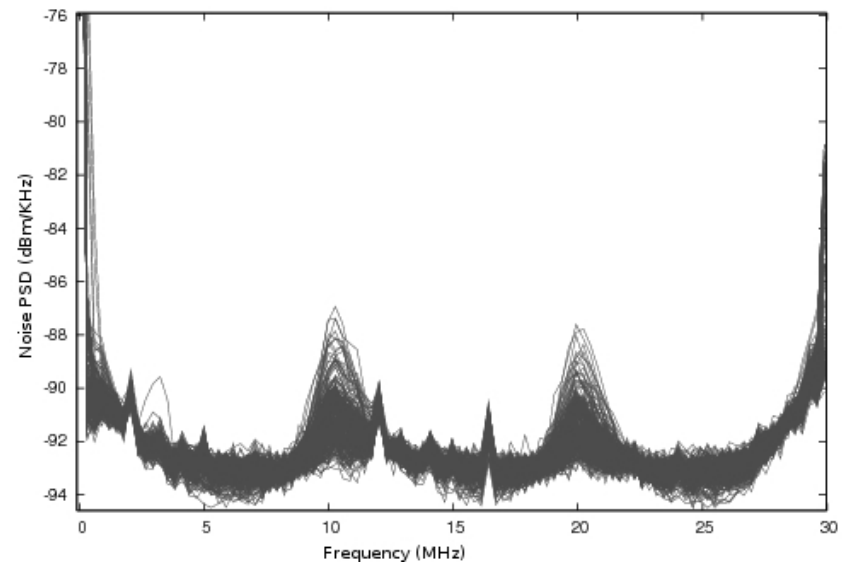

Fig. 8. Variant channel generation test results: noise PSD at the receiver

(DFT) on them we can obtain the channel frequency response at every interval. Fig. 11 shows the channel frequency response corresponding to the invariance intervals 0 (left) and 150 (right), as obtained from the simulator output. A variation is appreciated, showing that the simulator effectively offers a different response at each interval.

On a Pentium IV at $2.4 \mathrm{GHz}$ with $256 \mathrm{MB}$ of memory, 10000 samples can be processed in about 45 " using the LPTV model with $L=292$ invariance intervals. Using the same model, it takes about 15 " per noisy load to obtain the channel response and $60 "$ to calculate the filter coefficients.

\section{CONCLUSION}

An indoor power-line channel simulation tool has been presented. The subjacent behavioral model, constructed from a model of the physical structure of the network, includes both the frequency dependence and the short- and long-time variation of the channel. The calculation of the frequency response of the channel and the spectral distribution of the noise at the receiver end, the channel generation, is based on 


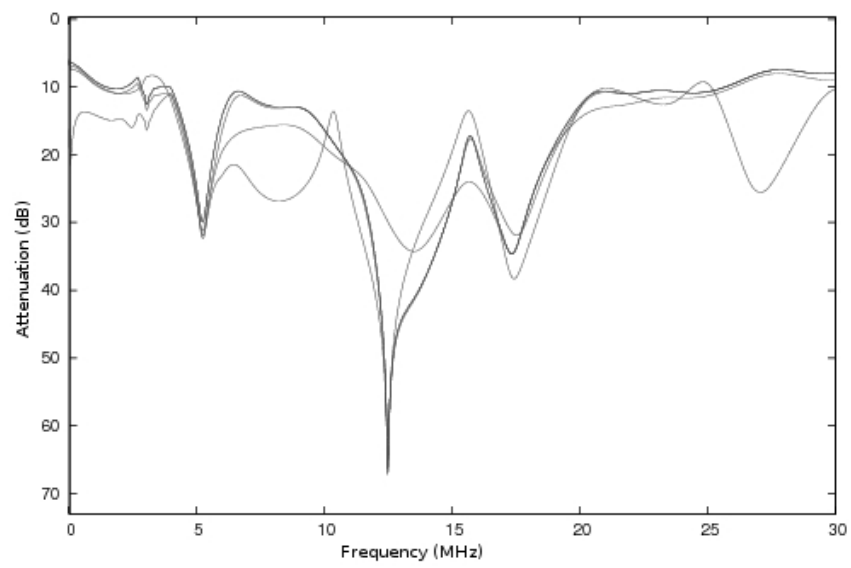

Fig. 9. Dynamic channel generation test results: channel response amplitude

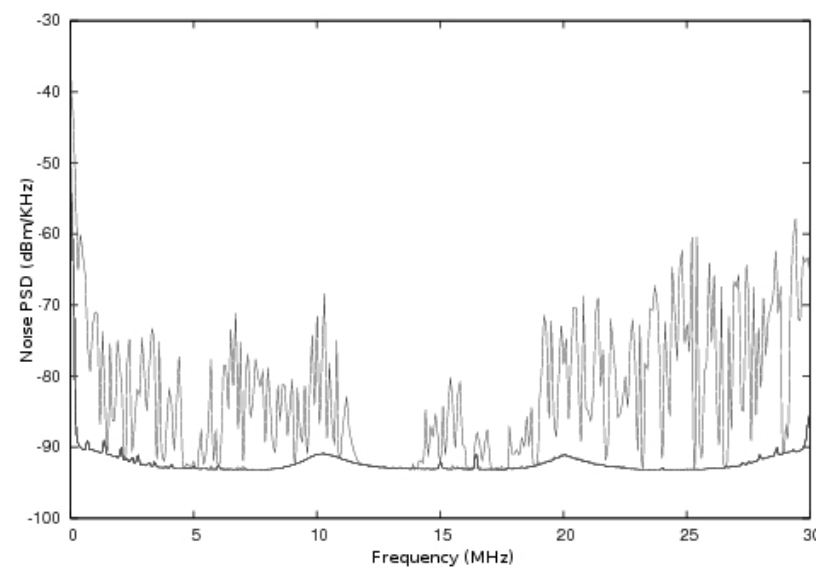

Fig. 10. Dynamic channel generation test results: noise PSD at the receiver

a structure of terminated lossy transmission lines. Afterwards, the channel simulation is carried out by means of two variablesize banks of LTI filters, one of which emulates the channel response and the other colors a white noise signal.

The simulator offers numerous applications. With a minimum knowledge of the network to be simulated, namely the number of electrical circuits and how large the home is, it is possible to generate channel models whose characteristics will match those of the ones a modem will face. The channel generation procedure allows for a statistical analysis of the indoor power-line channel behavior, using the data obtained from a number of plausible network topologies generated at random. Besides, the channel sensitivity in relation to the network physical parameters- link distance, wiring nature and structure, load impedance values, etc.-, can be statistically studied. On the other hand, the channel simulation procedure can be used to optimize transmission techniques like DMT modulation, the most popular one in PLC. With this target in mind, a server-mode simulation utility was implemented, in which two processes- transmitter and receiver- connect to the simulator using a network protocol. The simulation is then performed on a sample-by-sample basis, and the transmission parameters can be dynamically adapted. This mode can also be used to design reception techniques- equalization, synchro-
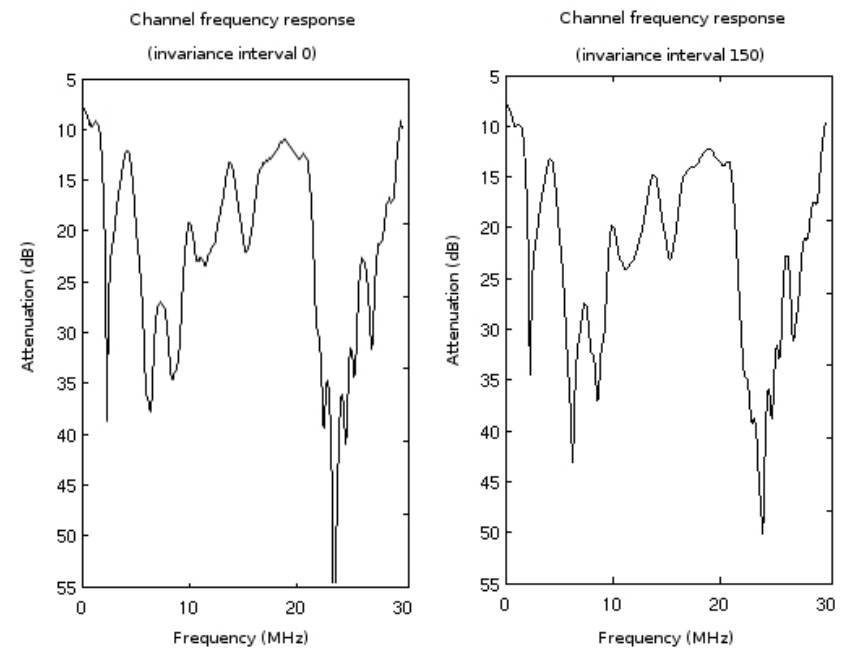

Fig. 11. Channel simulation test results

nization, etc. State-of-the-art modems for PLC are so flexible at managing the channel time and frequency selectivity that the goal is to simulate/emulate just realistic channels, neither statistical nor deterministic ones.

Although the simulator was written using the $\mathrm{C}$ programming language, the same approach can be used to create an FPGA-based real-time channel emulator.

\section{REFERENCES}

[1] F.J. Cañete, J.A. Cortés, L. Díez and J.T. Entrambasaguas, "Analysis of the Cyclic Short-Time Variation of Indoor Power-line Channels", IEEE Journal on Selected Areas in Communications, Volume 24, Issue 7, pp. 1327-1338, July 2006

[2] H. Philipps, "Performance Measurements of Power-line Channels at High Frequencies", Proc. 2nd International Symposium on Power Line Communications and Its Applications, pp. 229-237, ISPLC, 1998.

[3] F.J. Cañete, J.A. Cortés, L. Díez and J.T. Entrambasaguas, "Modeling and Evaluation of the Indoor Power Line Transmission Medium", IEEE Communications Magazine, pp. 2-8, April 2003.

[4] M. Katayama, T. Yamazato, H. Okada, "A Mathematical Model of Noise in Narrowband Power Line Communication Systems", IEEE Journal on Selected Areas in Communications, Volume 24, Issue 7, pp. 1267-1276, July 2006.

[5] S. Katar, B. Mashburn, K. Afkhamie, H. Latchman and R. Newman, "Channel Adaptation Based on Cyclo-Stationary Noise Characteristics in PLC Systems", IEEE International Symposium on Power Line Communications and Its Applications 2006, pp. $16-21$.

[6] M. Zimmermann and K. Dostert, "Analysis and Modeling of Impulsive Noise in Broad-band Powerline Communications", IEEE Transactions on Electromagnetic Compatibility, pp. 249-258, Feb. 2002.

[7] F.J. Cañete, L. Díez, J.A. Cortés, J.T. Entrambasaguas, "Broadband Modelling of Indoor Power-line Channels", IEEE Transactions on Consumer Electronics, pp. 175-183, 2002

[8] H. Philipps, "Modelling of Power Line Communication Channels", in International Symposium on Power-Line Communications and its Applications. pp. 14-21, ISPLC, 2005.

[9] M. Zimmerman and K. Dostert, "A Multipath Model for the Power-line Channel", IEEE Trans. on Communications, pp. 553-559, Apr 2002.

[10] J.G. Proakis, "Digital Communications", McGraw-Hill, 1995.

[11] L. Kleinrock, "Queuing Systems, vol. 1: Theory", Willey, 1975.

[12] W. Gardner, "Introduction to Random Processes", MacMillan, 1986.

[13] F.J. Cañete, "Caracterización y Modelado de Redes Eléctricas Interiores como Medio de Transmisión de Banda Ancha". PhD Thesis. 2005.

[14] J. Proakis and D. Manolakis, "Digital Signal Processing (3rd ed.): Principles, Algorithms and Applications ”, Prentice Hall, 1998. 